See discussions, stats, and author profiles for this publication at: https://www.researchgate.net/publication/345996905

\title{
A Visual Intelligence Scheme for Hard Drive Disassembly in Automated Recycling Routines
}

Conference Paper - November 2020

DOI: 10.5220/0010016000170027

CITATIONS

7 authors, including:

48

Erenus Yildiz

Georg-August-Universität Göttingen

4 PUBLICATIONS 2 CITATIONS

SEE PROFILE

67. Erwan Renaudo

University of Innsbruck

16 PUBLICATIONS 62 CITATIONS

SEE PROFILE

Some of the authors of this publication are also working on these related projects:

ReconCell View project

Project

Input correlation learning (ICO) View project

\section{READS}

82

Tobias Brinker

Leibniz Universität Hannover

1 PUBLICATION 0 CITATIONS

SEE PROFILE

Simon M. Haller-Seeber

University of Innsbruck

10 PUBLICATIONS 90 CITATIONS

SEE PROFILE 


\title{
A Visual Intelligence Scheme for Hard Drive Disassembly in Automated Recycling Routines
}

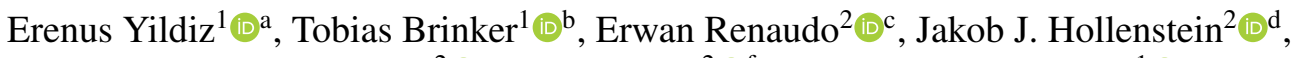 \\ Simon Haller-Seeber ${ }^{2} \mathbb{1}^{\mathrm{e}}$, Justus Piater ${ }^{2} \mathbb{1}^{\mathrm{f}}$ and Florentin Wörgötter ${ }^{1}{ }^{\mathrm{g} g}$ \\ ${ }^{1}$ III. Physics Institute, Georg-August University of Göttingen, Germany \\ ${ }^{2}$ Department of Computer Science, University of Innsbruck, Innsbruck, Austria
}

Keywords: Object Detection, Object Classification, Automation, e-Waste, Recycling.

\begin{abstract}
As the state-of-the-art deep learning models are taking the leap to generalize and leverage automation, they are becoming useful in real-world tasks such as disassembly of devices by robotic manipulation. We address the problem of analyzing the visual scenes on industrial-grade tasks, for example, automated robotic recycling of a computer hard drive with small components and little space for manipulation. We implement a supervised learning architecture combining deep neural networks and standard pointcloud processing for detecting and recognizing hard drives parts, screws, and gaps. We evaluate the architecture on a custom hard drive dataset and reach an accuracy higher than $75 \%$ in every component used in our pipeline. Additionally, we show that the pipeline can generalize on damaged hard drives. Our approach combining several specialized modules can provide a robust description of a device usable for manipulation by a robotic system. To our knowledge, we are the pioneers to offer a complete scheme to address the entire disassembly process of the chosen device. To facilitate the pursuit of this issue of global concern, we provide a taxonomy for the target device to be used in automated disassembly scenarios and publish our collected dataset and code.
\end{abstract}

\section{$1-$ INTRODUCTION}

Today's electronic products have a short life cycle, as they are usually discarded before their materials degrade. Studies like (Duflou et al., 2008) showed that 1) the recycling processes of these products are mostly done by humans, 2) even when automated, the processes are usually destructive - leading to the loss of valuable metals and rare earth materials (Tabuchi, 2010; Massari and Ruberti, 2013). There is thus a strong need for less-destructive recycling processes, as illustrated by a recent initiative to reuse magnets from retired hard-disk drives (HDD) in order to reduce the demand on rare earth materials ${ }^{1}$. In addition, the hazardous elements used in electronic products

\footnotetext{
a (D) https://orcid.org/0000-0002-3601-7328

b (D) https://orcid.org/0000-0003-2930-158X

c(1D) https://orcid.org/0000-0003-3282-8972

d (D) https://orcid.org/0000-0001-5694-691X

e (D) https://orcid.org/0000-0002-1538-5906

f(i) https://orcid.org/0000-0002-1898-3362

g (iD https://orcid.org/0000-0001-8206-9738

${ }^{1}$ https://spectrum.ieee.org/energywise/energy/environment/
} making-new-hard-disk-drives-from-recycled-magnets (e.g. Beryllium in GSM amplifiers) pose a threat to the health of employees due to their carcinogenic nature. Even with enforced security measures, avoiding exposure of humans to such substances is paramount. Thus, there are both health and economical reasons to improve automation of the disassembly and recycling processes.

From a robotics perspective, the disassembly process presents a high automation potential due to its repetitiveness. However great adaptability of such a system is required, as EOL products to be recycled can be of various nature (from house-office equipment to entertainment, communication, etc.) or they might be partially damaged. Moreover, some devices within the same family of products present a high intra-class variance of their parts' shape depending on their brand or model. These variances implies that a robotic disassembly system can not rely on an open-loop procedure but has to analyze the product to find the best disassembly strategy. It thus needs efficient perception capabilities to provide relevant information to the rest of the system. Several parameters must be extracted from the scene: product's parts must be identified from the sensor data 
and their pose must be estimated to allow the robot to interact with the device. Sensors of various types can be used (precise laser scanner, etc. (Weigl-Seitz et al., 2006)), however vision sensors offer the best cost/performance/adaptability solution given the tasks to complete. In this work, we thus introduce a visionbased perception scheme capable of analyzing a product at different stages of disassembly. We evaluate it on one class of undamaged devices, computer hard drives (HDDs), which are one of the most common devices encountered in E-Waste, and suitable in size for robotic manipulation. This paper is organized as follows: Section 2 gives a review of the literature on vision-based methods, Section 3 presents our approach in detail, Section 4 presents the evaluation of the method on the target device. Section 5 discuss the impact of these results and concludes.

\section{RELATED WORK}

Computer vision techniques have been extensively applied to industrial applications, e.g. defect detection, part recognition, etc. (Bi and Wang, 2010; Malamas et al., 2003). However, not much work is done in the context of automated recycling, where multiple valuable parts must be identified for recovery. A short survey of existing approaches in 2006 (WeiglSeitz et al., 2006) showed numerous limitations also found in later work. First the focus on detecting a specific class of component to detect - e.g. screws ((Bdiwi et al., 2016; Ukida, 2007; Bailey-Van Kuren, 2002), bolts (Wegener et al., 2015) - reduces the generality of the method and its applicability in the context of recycling where several types of components must be gathered. The use of fully modelbased methods (Elsayed et al., 2012; Xie et al., 2008; Ukida, 2007; Pomares et al., 2004; Torres et al., 2003; Tonko and Nagel, 2000) implies the availability of (e.g. CAD) models. This is a strong assumption as these models are rarely available for every brand and every type of device. Moreover, the recycling domain does not guarantee that devices are in an intact state. Finally, another important limitation is the use of non-adaptive feature detection techniques (Bdiwi et al., 2016; Wegener et al., 2015; Weigl-Seitz et al., 2006; Bailey-Van Kuren, 2002; Karlsson and Järhed, 2000). As mentioned previously, the high intra-class and inter-brand variances of components reduces the relevance of a human-engineered descriptor of a component class. This limitation is only partially explained by the date of publication of some works (Weigl-Seitz et al., 2006; Bailey-Van Kuren, 2002; Karlsson and Järrhed, 2000).
On one hand, it seems that there is still a substantial lack in generalizable, device and environmentindependent methods, which can be used in disassembly processes. On the other hand, Deep Convolutional Neural Networks (DCNN) offer a powerful solution to analyze the inner structure of devices in the context of disassembly. These methods have the potential to solve the problems highlighted before: the specificity of a detector can be tackled by training on a dataset that includes samples of multiple parts of the targeted device (and extended by retraining later on) and thus being able to recognize these parts in all the devices that use them. The lack of adaptability is addressed by the nature of deep networks: as machine learning methods, they are problem and feature agnostic before training. Finally most of these methods are CAD model-free, meaning that they do not require device-specific description sheets. They learn the relevant features of a part class by themselves, abstracting from the details of a specific brand and model. In E-Waste recycling context, devices found in the same family of devices often include similar parts such as PCBs, screws, wires, etc. Extracting features from these using visual paradigms usually leads to reproducible results that can be used and further developed for other devices as well.

Thus, the problem of disassembly scene analysis can be formulated as a problem where machine learning paradigms (e.g. segmentation, classification) and traditional data (e.g. pointcloud) analysis methods are used in order to recognize the present parts of the target device. In contrast to other application domains, the context of recycling E-Waste adds the following constraints:

- The position estimation of parts must be precise enough to allow manipulation of small parts in the device.

- There is a strong degree of occlusion because parts are intertwined, e.g. the platters and $\mathrm{R} / \mathrm{W}$ head of a hard drive.

- There is substantial intra-class variance for specific parts depending on the device brand or model or the potential damage of the part.

The Semantic Segmentation problem (also known as pixelwise classification problem) has been addressed in domains ranging from autonomous driving, human-computer interaction, to robotics, medical research, agriculture (Li et al., 2017; Dai et al., 2016; Liu et al., 2018; Fu et al., 2019; Lin et al., 2019; Dvornik et al., 2017). However, to the best of our knowledge, no work currently address the domain of automated recycling of E-Waste. In these domains, the state-of-the-art performance is achieved 
using deep learning methods, especially Convolutional networks (CNNs or ConvNets) (Krizhevsky et al., 2012). Fully Convolutional Networks (FCN) have been the standard algorithm to achieve pixelwise segmentation of images (Badrinarayanan et al., 2017; Long et al., 2015; Ronneberger et al., 2015) in various domains such as medicine, autonomous driving, domestic robotics. They extend CNN by replacing fully-connected layers by convolutional layers, allowing for arbitrary-sized input with no need for regionproposal. The trade-off however, due to the nature of the layers, is that the prediction of boundaries lacks precision. This problem is addressed by a set of improvement to the networks (e.g. multi-resolution architecture with skip connections (Ghiasi and Fowlkes, 2016; Lin et al., 2017a), mask refinement (Pinheiro et al., 2016)). Additionally, pixelwise segmentation requires labeled data which has a higher cost of production (Lin et al., 2014). Weakly-supervised methods have been developed to tackle this issue (Zhang et al., 2019): these methods iterate between learning using coarse bounding boxes as labels then refining them into more precise masks (Dai et al., 2015; Khoreva et al., 2017). They achieve similar performance as fully supervised methods at a lower labelling cost. Another main approach is called RegionBased Semantic Segmentation. This method relies on a pre-processing of the input image into candidate objects (region proposals) for which features are extracted. These features are then used for the classification. There is an entire family of R-CNN networks (Girshick et al., 2014; Girshick, 2015; Ren et al., 2015) that have been evolving and finally the current state-of-the-art networks of the family is called Mask-RCNN (He et al., 2017) which is based on Feature Pyramid Network (FPN) (Lin et al., 2017b). Mask-RCNN has been used by Facebook AI Research (Joulin et al., 2016) as well as by medicine studies (Johnson, 2018; Couteaux et al., 2019; Chen et al., 2019).

Mask-RCNN and its ensembled models are currently the state of the art for object detection and instance segmentation, and the reason for this is the region-based detection mechanism mentioned earlier. The core idea behind Mask-RCNN is to scan over the predefined regions called anchors. RPN, then, does two different types of predictions for each anchor. First is the score of the anchor being foreground, and the bounding box regression. The fixed number of anchors with the highest score are then chosen, and the regression adjustment is applied to get the final proposal for object prediction at the network head. There are other architectures that could be used for this very task, such as the ensembled model Cascade-
RCNN (Cai and Vasconcelos, 2018). Through the experimental evaluation we conducted in Section 4, we found out that Mobile-RCNN (Howard et al., 2017) works better for our domain.

\section{METHODS}

Our visual scheme outputs on request a scene analysis that combines the predictions of 3 modules: a part detection module, a screw detection module and a gap detection module. The part detection module recognizes most inner components of electronic devices (HDD in our case). A specialized screw detector module detects various screw types as a separate task from the part detection. Finally, gaps are also detected as they provide useful information for robotic manipulation of parts and tool. The pseudo code for the pipeline is given in algorithm 1 .

As mentioned earlier, the analysis of parts is a semantic segmentation problem, but the screw detection can be simplified as a classification problem: rather than trying to find screws boundaries, we make the hypothesis that they are mostly circular structures in the scene. The new problem is thus to classify detected circles in the image as screws or artefacts (holes, stickers, etc.), with a specialized network.

\subsection{Taxonomy \& Datasets}

In order to train the different modules of our pipeline, we collect and create datasets. The first step is to define a taxonomy of the HDD, which is done in agreement with an industrial partner providing hardware samples and data. The taxonomy provides the class labels and is available on the IMAGINE website $^{2}$. Two datasets are created (a) for parts and (b) for screws. For (a), top-down views of the computer hard drives are annotated into 12 classes corresponding to parts of interest according to the taxonomy. 500 images of 7 different brands (Hitachi, IBM, Maxtor, Seagate, WD, Samsung, Toshiba) and models are collected under different light conditions. These images include various stages of disassembly with parts in arbitrary positions (e.g. after a failed action). $5 \%$ of all images correspond to damaged devices to account realistically for the state of products in recycling plants. The dataset is split into a validation set $(10 \%$ of the images), a test set (10\%) and a training set (80\%). For (b), 10700 samples are annotated into $23 \%$ of screws and $77 \%$ of artifacts. The training set includes 1491 screws and 4924 artifacts. The test set includes

${ }^{2}$ https://imagine-h2020.eu/hdd-taxonomy.php 


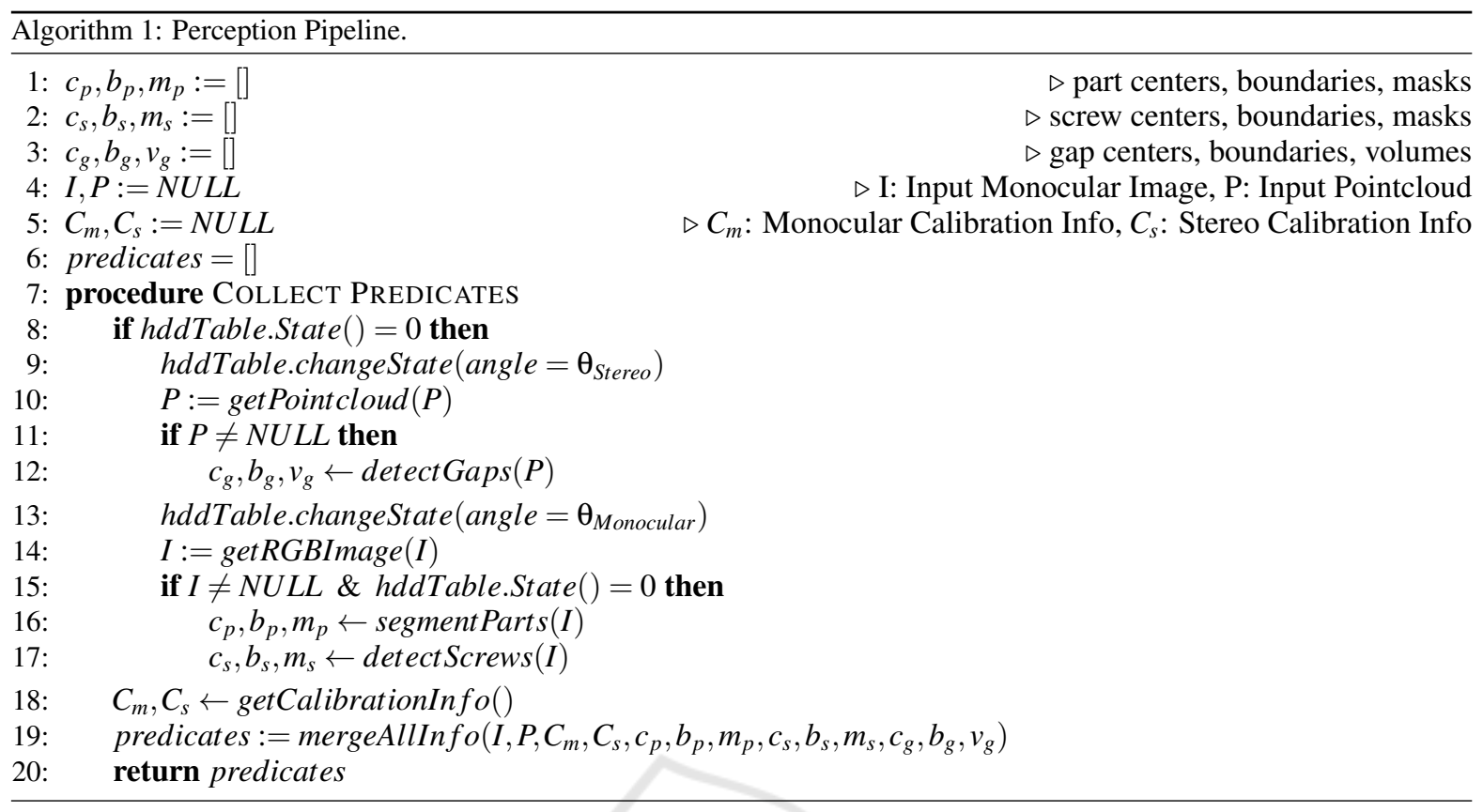

1000 screws and 3285 artifacts. Figure 1 illustrates an annotated drive (top) and a sample of artefacts and screws found during the disassembly (bottom).

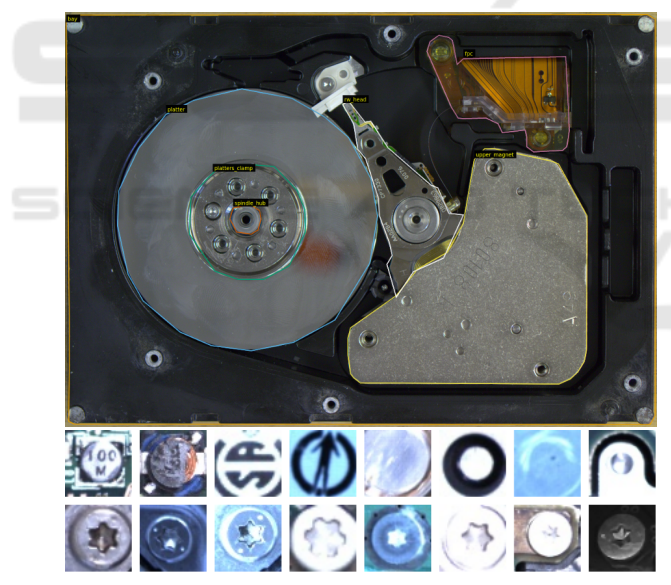

Figure 1: Top: Ground truth annotations for a sample hard drive based on the taxonomy, used for part segmentation task (above). Bottom: Training dataset containing (first row) artefacts and (second row) screws for the detection of screws.

\subsection{Part Segmentation}

We address the semantic segmentation problem with Deep CNNs to detect, recognize and localize the inner parts of the device. Due to the high number of available state-of-the-art networks, we train and evaluate a set of selected methods on this instance segmentation problem: MobileNetV1 (Howard et al., 2017), MobileNetV2 (Sandler et al., 2018), Cascade-RCNN
(Cai and Vasconcelos, 2018), native Mask-RCNN (He et al., 2017), and finally the ensembled model of Cascade-MobileNetV1 . All networks were trained with data augmentation turned on as suggested by the native Mask-RCNN authors, we apply the same augmentation procedures. The accuracy of a network is computed using the similarity $I o U$ metric (see eq. 1):

$$
I o U=\frac{|A \cap B|}{|A \cup B|}
$$

where $\mathrm{A}$ and $\mathrm{B}$ are respectively the sets of predicted and ground truth bounding boxes. The results of this preliminary study are available in Table 1. Although Cascade-RCNN obtains the best segmentation results, we selected Mobile-RCNN that comes right after, since the model is lighter, and, thus, it is employed in the part detection module of our architecture.

\subsection{Screw Detection}

The screw detection module classifies potential candidates input into the screw or artefact classes. We integrate the algorithm presented in (Yildiz and Wörgötter, 2019) into our pipeline. We describe the algorithm briefly hereafter and refer the reader to the publication for an extended description and analysis.

The algorithm combines classical vision preprocessing of the input image with deep learning methods for classification. The image is processed with the Hough circle transform (Duda and Hart, 1971) to generate screw candidates as local views of the in- 
Table 1: IoU values of different networks evaluated on our test set. Cascade-RCNN and MobV1-RCNN seem to be the best performing ones.

\begin{tabular}{|c|c|c|c|c|c|c|c|c|c|}
\hline DCNN Identifier & Cascading & Backbone & $A P$ & $A P_{0.5}$ & $A P_{0.75}$ & $A P_{s}$ & $A P_{m}$ & $A P_{l}$ & Mean-F \\
\hline Mask-RCNN & No & ResNet-101-FPN & 41.2 & 65.7 & 47.6 & 16.3 & 42.3 & 42.4 & 0.70 \\
\hline MobV1-RCNN & No & Mobilenetv1-224-FPN & 56.2 & 76.7 & 64.9 & 20.6 & 54.6 & 59.7 & 0.78 \\
\hline MobV2-RCNN & No & Mobilenetv2-FPN & 34.6 & 60.5 & 35.5 & 3.8 & 15.4 & 43.6 & 0.63 \\
\hline Cas-Mob-RCNN & Yes & Mobilenetv1-224-FPN & 28.5 & 43.3 & 33.3 & 20.0 & 13.3 & 35.4 & 0.61 \\
\hline Cas-RCNN & Yes & ResNet-101-FPN & 61.3 & 76.4 & 72.6 & 35.1 & 61.8 & 65.4 & 0.84 \\
\hline
\end{tabular}

put image. These candidates are sent to an ensemble of two networks (InceptionV3 (Szegedy et al., 2016) and Xception (Chollet, 2017) both with an additional dropout layer before their last fully-connected layer) and their output are combined through a weighted sum. The network finally output its prediction on the nature of the candidate (screw or artifact). The method is reported to achieve a $99 \%$ successful classification rate (Yildiz and Wörgötter, 2019).

The most significant feature of this work is clearly its adaptation ability. Since we are proposing a visual scheme for disassembly processes, we must consider the fact that our target device (HDD) is not going to be the only device to be disassembled for long. Since the chosen screw detection paradigm already works well for the screws encountered in hard drives, along with the fact that it allows users to collect new datasets very quickly, we are sure of its adaptability even if the target device or the environment changes at a point.

We've experimented with the integrated screw detection module and we share its results in Section 4.

\subsection{Position Estimation}

The part detection and screw detection module operate only on RGB image data. To act on the detected screws and parts, their pixel coordinates (2D) need to be related to positions in the robot's frame (3D). We will refer to this process as localization. We use an RGB-D camera to get a point-cloud representation of the scene and relate pixels to points (see Figure 3 for the relative poses of the camera). The correspondence is done by projecting the point-cloud points into pixel coordinates, and estimating a k-nearest-neighbor regressor to perform the inverse operation: mapping pixels to 3D positions. This process is repeated each time the scene analysis is performed, i.e. upon request from the rest of the system. These requests come asynchronously at less than $1 \mathrm{~Hz}$ thus there are no time constraint in this case.

\subsection{Gap Detection}

The gap detection relies on the top-view point-cloud representation of the scene acquired by the stereo camera. The overall process along sample views for each step is presented in Figure 2. The point-cloud is processed by a passthrough filter to find the region of interest: points outside a volume (i.e. boxshaped) in which the HDD should be positioned are discarded to remove artifacts. The output point-cloud is then denoised: We are using sparse outlier removal (Rusu et al., 2008), which identifies outlier points based on their mean distance to their nearest neighbours. The denoised point-cloud depth distribution is then analysed to classify the points into surface points and gap points. Because the existence of gaps produces a bi-modal depth distribution between foreground points (surface) and background points (gaps), a simple threshold can be computed automatically (e.g. using (Otsu, 1979)). The candidate gap points are then clustered into separate gaps using a densitybased clustering approach (DBSCAN with manually set parameters). This step provides the location and size of the gaps and excludes the spaces too small to be interesting gaps. In order to extrapolate the volume of each gap, a convex hull algorithm is used to get the $2 \mathrm{D}$ boundaries of the gap in a first pass. Then artificial points are added to each cluster by duplicating the boundary points and setting their height to the median height of the surface points identified earlier. A second pass of the convex hull algorithm provides the corrected 3D gap volumes, which are then filtered to remove very small volumes. The gap centers are estimated as the mean of the extrema for every axis. The set of found gaps including their centers and volumes are sent back to the rest of the pipeline.

\section{RESULTS}

\subsection{Experiments}

\subsubsection{Setup}

Our visual scheme requires two inputs: a top-down RGB image and a top-down point-cloud. We built an automated system that uses a tilting table holding the device either horizontal (its surface normal aligned with the RGB camera) or $45^{\circ}$ (aligned with the RGB- 


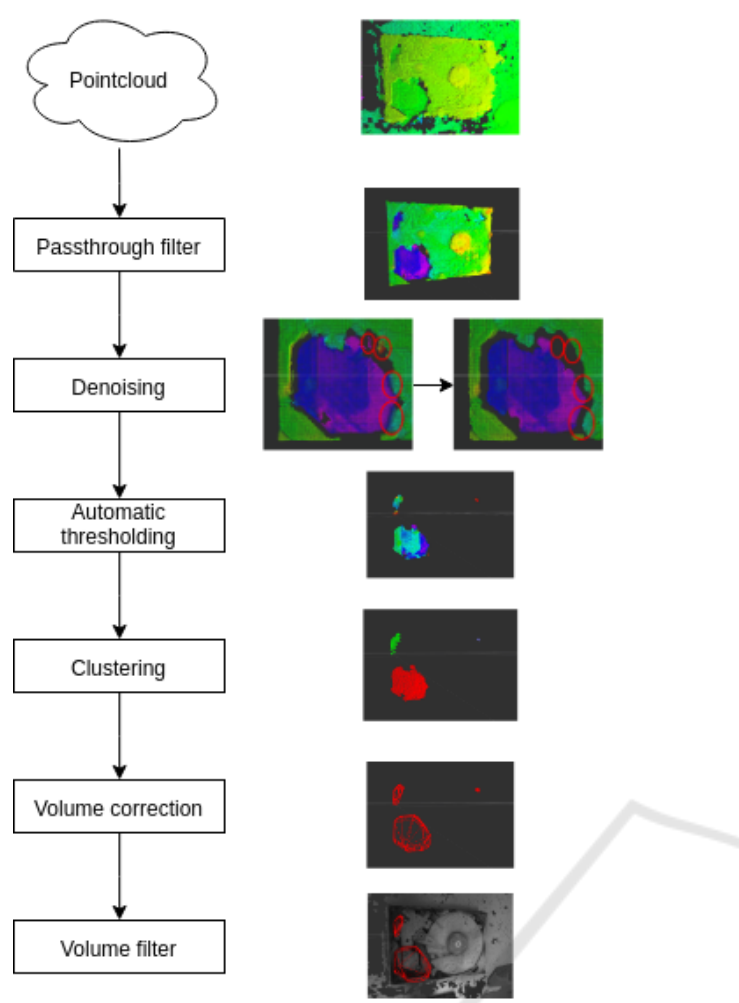

Figure 2: Complete pipeline for the gap detection module. Initially, a passthrough filter discards the points below the lower limit and points above the upper limit for the specified axis. The noise created from the stereo cameras is removed via sparse outlier removal (Rusu et al., 2008). Following, thresholding is done only on the Z-axis since it denotes the height of the points in the cloud. Deeper points are very likely to belong to a gap, therefore, using a well-chosen threshold we can split the point cloud based on the depth of points into potential gap points and insignificant surface points. Points belonging to a gap often lie in close proximity to each other, opening an opportunity for a clustering approach (DBSCAN, HDBSCAN) to label points. A volume correction is applied by adding artificial points to the set of gap points, so that the convex hull algorithm uses them as the new outline points and creates a better estimate. As a last step, the volume filter is used to throw away extremely small volumes that cannot be gaps given the device's natural shape.

D stereo camera) on request (see Figure 3). We use a Basler acA4600-7gc monocular camera which provides images with $4608 \times 3288$ resolution and 3.5 FPS and a Nerian Karmin2 stereo camera with a depth error of $0.06 \mathrm{~cm}$ from the minimum range of $35 \mathrm{~cm}$.

\subsection{Evaluation Method}

There are three main blocks in our scheme and each of these blocks has to be evaluated differently, as the paradigms running behind are different. Evaluation of the screw detection used was already conducted in

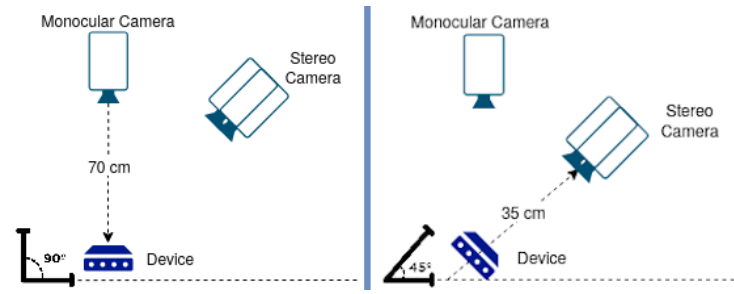

Figure 3: Setup used for evaluation: a monocular RGB camera is oriented downwards $\left(\theta_{\text {Monocular }}=90^{\circ}\right)$ and a stereo RGB-D camera is mounted at $\theta_{\text {Stereo }}=45^{\circ}$. This configuration is due to the different focal lengths of the cameras. Both are pointing at the device to be analysed. A tilting mechanism allows the system to obtain "top-down" views of the devices with both cameras.

its paper, thus, the results are directly taken from that work (Yildiz and Wörgötter, 2019). For part segmentation, we are going to go by the IoU (Intersection over Union) metric and report the AP, as it has become the de facto evaluation metric for instance segmentation networks. One very similar work was recently evaluated the same way (Jahanian et al., 2019). Finally the gap detection is evaluated with a metric that we came up ourselves, which we are going to discuss in its respective subsection.

\subsubsection{Screw Detection}

Table 2: Classifier accuracy of different state-of-the-art networks, from (Yildiz and Wörgötter, 2019) with permission.

\begin{tabular}{|l|l|l|l|l|l|}
\hline \multicolumn{1}{|c|}{ Model } & TP & TN & FP & FN & Acc \\
\hline Xception & 975 & 3244 & 25 & 41 & 98.5 \\
\hline InceptionV3 & 973 & 3262 & 27 & 23 & 98.8 \\
\hline ResneXt101 & 962 & 3260 & 38 & 25 & 98.5 \\
\hline InceptionResnetV2 & 965 & 3260 & 35 & 25 & 98.6 \\
\hline DenseNet201 & 897 & 3272 & 103 & 13 & 97.3 \\
\hline ResNetV2 & 943 & 3208 & 57 & 77 & 96.9 \\
\hline Integrated model & $\mathbf{9 8 8}$ & $\mathbf{3 2 5 4}$ & $\mathbf{1 2}$ & $\mathbf{3 1}$ & $\mathbf{9 9 . 0}$ \\
\hline
\end{tabular}

We first would like to report the classifier accuracy in Table 2 which are directly taken from the work (Yildiz and Wörgötter, 2019) published. However, although the classifier accuracy is quite high, due to the fact that Hough circle finder method misses out finding the circles in the first place, our final average precision was found to be $80 \%$. As the classifier expects images that are directly suggested by the Hough circle finder, any circle that is missed, is also not considered by the classifier. In other words, the classifier's ability is limited by the Hough circle finder. Therefore, the AP remains at $80 \%$. We refer the reader to the Figure 4 to illustrate a few detection samples during the HDD disassembly sequences. 


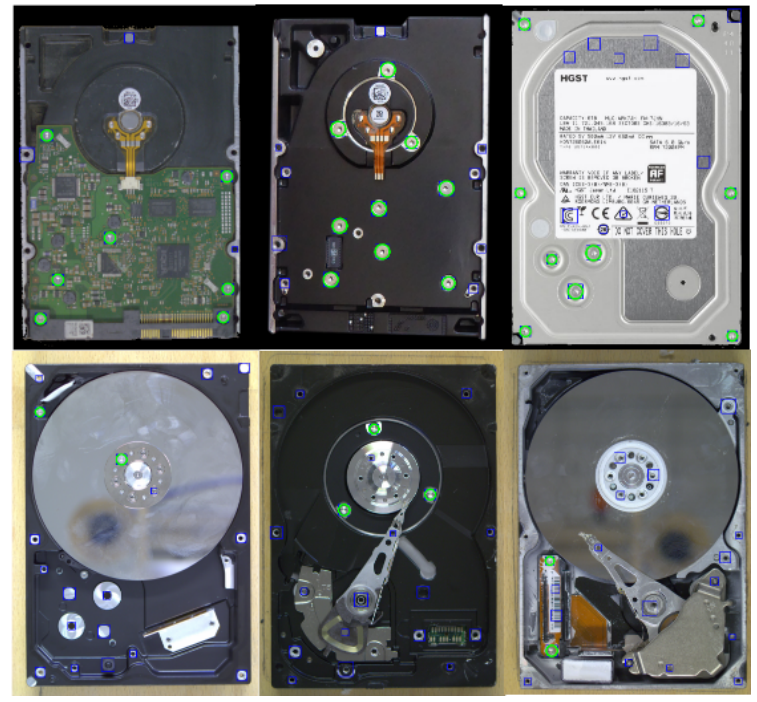

Figure 4: Outputs from the integrated screw detection module. Blue circles highlight the candidates suggested by the Hough circle finder, whereas green circles are the detected screws by the model taken from a study (Yildiz and Wörgötter, 2019).

\subsubsection{Part Segmentation}

As mentioned earlier, the standard metrics for pixel to pixel segmentation are mainly the COCO (Lin et al., 2014) average precision (AP) metrics: $A P$ is average precision of multiple IoU values. As reported in Table 1, MobV1-RCNN and CAS-RCNN achieve the best segmentation performances (resp. 0.78 and 0.84 ). These results are achieved after 500 epochs of training on Google Colaboratory (Bisong, 2019) environment using Tensorflow (Abadi et al., 2016) 1.15.2. Figure 5 shows a sample output of the part segmentation process.

From our experiments, we make the following conclusions. MobileNetV2 has stabilization issues since the model is not able to predict on every scene after a certain amount of training. We observed in the training history that the loss becomes undetermined and thus the model remains unstable. It could be argued that the reason is the dataset, i.e. the classes are not uniformly distributed. On the other hand, cascading lighter backbones such as MobileNetV1 does not ensure better feature extraction property. In fact, our modeling suggests that it actually decreases efficiency due to usage of depthwise convolutions. However, this situation in return, validates the choice of backbone with residual nets that is clearly done in the original CBNet (Liu et al., 2019), Cascade-RCNN (Cai and Vasconcelos, 2018). In short, we find out that cascading should only be done with models that has residual backbones such as ResNet and its variants.

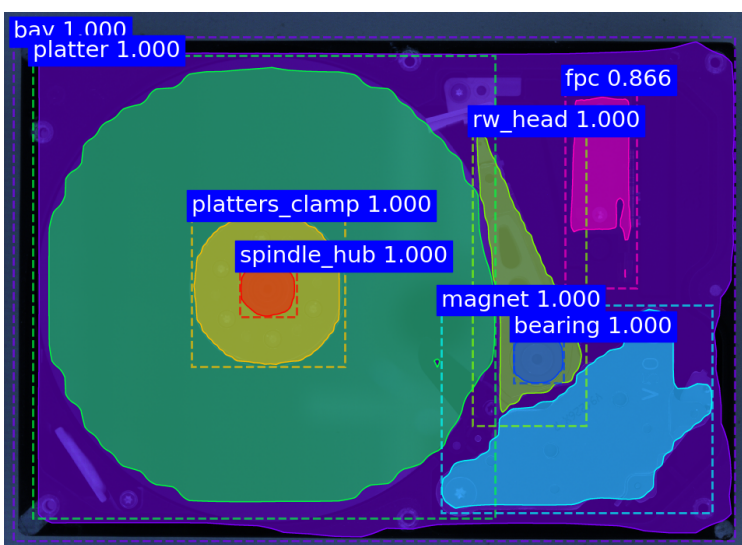

Figure 5: Output of the part segmentation module powered by MobV1-RCNN. Each color represents a different part predicted by the network whose names and prediction values are given. In this case, all parts and boundaries are correctly detected.

During our investigations we noticed that increasing cascading with more detection target and logits loss destabilizes the model. The use of DenseNet (Huang et al., 2016) may increase accuracy, however, with non-distributed class data like ours, stabilization remains an issue. This is due to the fact that the last layers in the network cannot handle class-logits when the feature data is non-distributed. In the presence of multi-GPU systems, we recommend ResNext-152 (Xie et al., 2017) to be investigated for higher accuracy.

Instead of selecting Cascade-RCNN, we made our choice with MobV1-RCNN due to the fact that it remains stable in the presence of high resolutions, unlike Cascade-RCNN that is unstable despite of having high accuracy. Since our setup uses high-resolution images, we therefore employed MobV1-RCNN as our final model in the segmentation scheme. However, for future reference, we note that combining images of different resolutions in a dataset creates problems in stable models as the model is not stable during training.

\subsubsection{Gap Detection}

In order to evaluate the performance of the gap detector, we had to create ground truth. To this end, all point clouds taken by the stereo cameras were annotated using a Semantic Segmentation Editor ${ }^{3}$. These ground truth annotations consist of point wise segmentations of each gap in a device. To get comparable point numbers, sparse outlier denoising was run on the clouds before segmentation. Based on the found

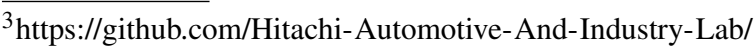
semantic-segmentation-editor
} 
Table 3: Comparison of the DBSCAN powered detector's output and the ground truth annotations. Mean error is found to be $24.30 \%$ among the identified gaps. Gaps that were not identified due to the shortcomings of the sensor were left out of the calculation. These are the gaps that are usually extremely small and irrelevant to the disassembly routine.

\begin{tabular}{|l|c|c|c|}
\hline & Detected $\left(\mathrm{cm}^{3}\right)$ & Ground Truth $\left(\mathrm{cm}^{3}\right)$ & Error(\%) \\
\hline Gap \#1 & 36.3 & 46.243 & 21.50 \\
\hline Gap \#2 & 3.099 & 3.417 & 9.31 \\
\hline Gap \#3 & 23.414 & 16.984 & 37.86 \\
\hline Gap \#4 & 22.714 & 22.712 & 0.01 \\
\hline Gap \#5 & 0.99 & 0.99 & 0.00 \\
\hline Gap \#6 & 0.725 & 0.746 & 2.82 \\
\hline Gap \#7 & 1.196 & 1.159 & 3.19 \\
\hline Gap \#8 & 8.854 & 8.032 & 10.23 \\
\hline Gap \#9 & 2.421 & 1.974 & 22.64 \\
\hline Gap \#10 & 16.622 & 15.99 & 3.95 \\
\hline Gap \#11 & 0.136 & 2.797 & 95.14 \\
\hline Gap \#12 & 26.874 & 36.339 & 26.05 \\
\hline Gap \#13 & 3.45 & 1.677 & 51.39 \\
\hline Gap \#14 & 12.043 & 12.663 & 4.9 \\
\hline Gap \#15 & 0.189 & 0.773 & 75.55 \\
\hline & & & Mean Error: 24.30 \\
\hline Gap \#16 & - & 0.125 & - \\
\hline Gap \#17 & - & 1.443 & - \\
\hline Gap \#18 & - & 0.386 & - \\
\hline Gap \#19 & - & 0.438 & - \\
\hline Gap \#20 & - & 0.453 & - \\
\hline Gap \#21 & - & 0.111 & \\
\hline
\end{tabular}

Table 4: Precision for the DBSCAN- and HDBSCANpowered gap detectors.

\begin{tabular}{|l|c|c|c|c|}
\hline & Detected & True positives & False positives & Precision \\
\hline DBSCAN & 18 & 15 & 3 & $\mathbf{8 3 . 3 3} \%$ \\
\hline HDBSCAN & 22 & 16 & 6 & $72.73 \%$ \\
\hline
\end{tabular}

points the volume correction was used to estimate a volume for the labelled gap. Given the estimated volume and the number of points for each gap in each device, the gap detector was used to process the same point cloud. For all devices the same set of parameters was used. Tuning these parameters on a device to device basis can improve the results and correct misclassifications. However, since the gap detector should have a degree of generalization, the parameters are fixed for every device. Additionally, the detector was used twice on each point cloud, once using DBSCAN in the clustering step of the approach and once using HDBSCAN. The detector outputs the volume and point numbers for each identified gap. These numbers are then compared against the human annotation. Table 3 reports the results. The entries of detection and error as "-" are the ones where our camera could not perceive the gap due to technical impossibilities given the hardware limitations of the camera and are not included in our calculations. The method achieved a mean error in volume of $24.30 \%$ among the identified gaps with a standard deviation of 28.14 and variance of 791.85 .

Furthermore, we evaluated our gap detector's clustering algorithm as well, since it plays a pivotal role in the pipeline. To this end, we considered the
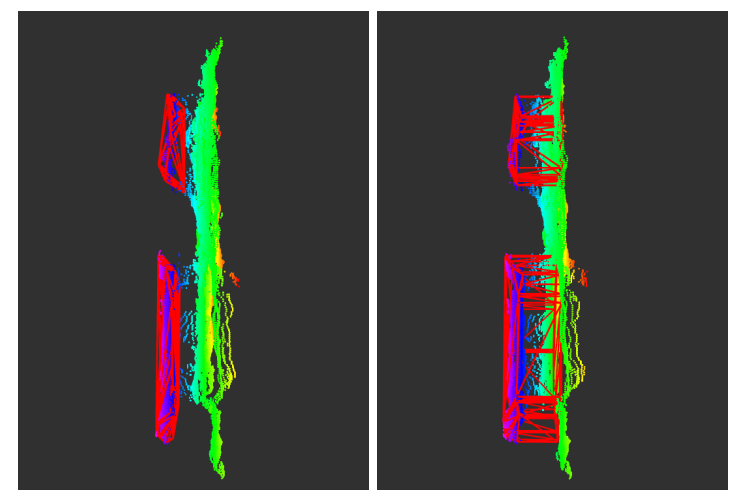

Figure 6: Detected gaps without (left) and with (right) volume correction.

two state-of-the-art clustering algorithms namely DBSCAN and HDBSCAN. In table 4 a detector based on DBSCAN is compared against the detector using HDBSCAN. Over the 12 hard drives used with a total of 21 gaps, the gap detector identified 18 gaps or $85.7 \%$ using DBSCAN. The detector based on HDBSCAN identified 22 gaps, a gap more than the ground truth. While using DBSCAN, the detector was able to identify 15 out of 21 gaps correctly against 16 out of 21 gaps using HDBSCAN. However, the higher detection rate of HDBSCAN also produces a higher number of false positives. The detection pipeline produced gaps with a precision of $72.73 \%$ using HDBSCAN against the $83.33 \%$ using DBSCAN. Furthermore, a detection pipeline using DBSCAN was able to produce higher quality gaps (see figure 6 for an illustration of the output of the gap detector). Therefore in our approach, a detection system using DBSCAN was preferable over a detection system using HDBSCAN.

\subsection{Generalization}

As mentioned earlier, our part segmentation model was trained with images that account for cases where the device is damaged or cases where a part falls back in the scene, occluding certain portion of a target device. These are significant cases to assess the generalization ability of the scheme. We are quite optimistic as our results for such cases prove that the scheme does generalize. Figure 7 illustrates a few cases of such.

\section{DISCUSSION}

In this paper, we presented an integrated vision pipeline to analyze an industrial scene and extract the composition of parts inside a device. We pro- 


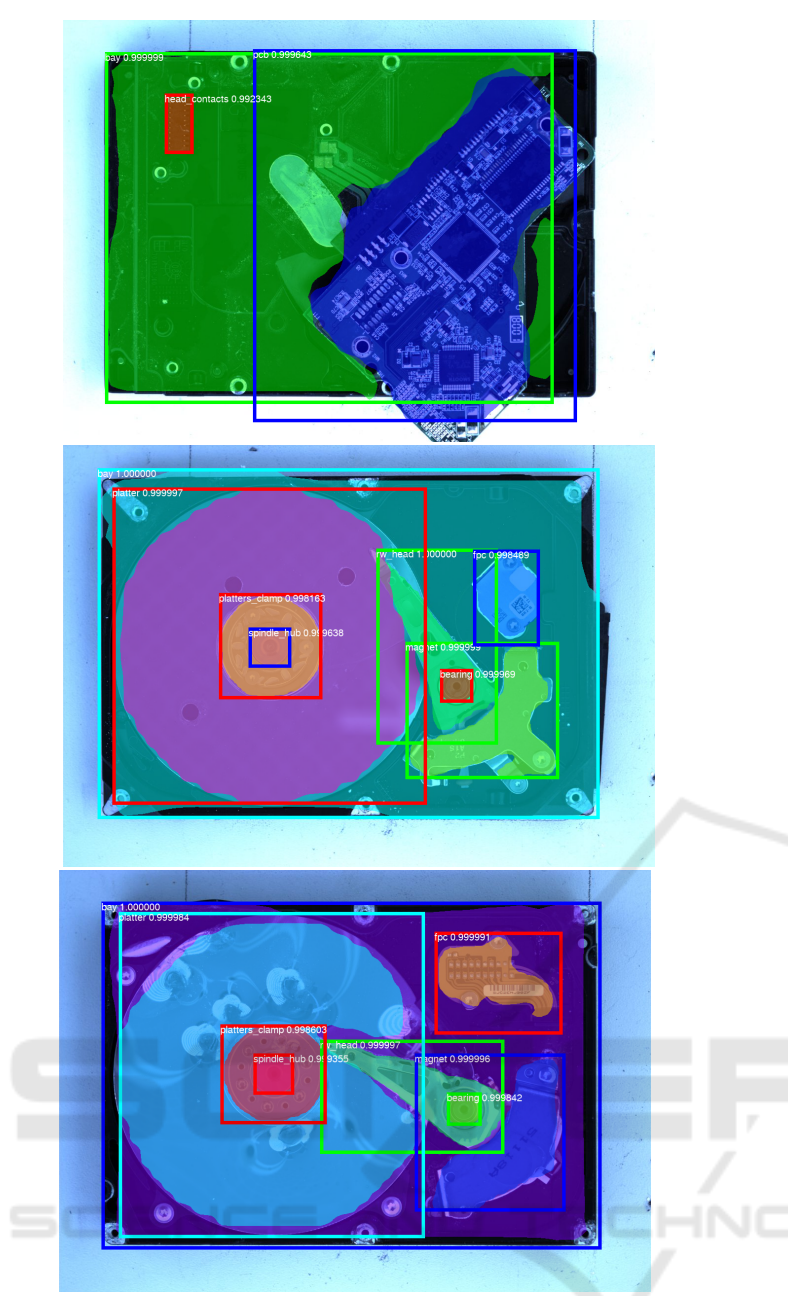

Figure 7: Output of the part segmentation module powered by MobV1-RCNN on damaged devices, as well as on devices where a part is arbitrarily placed over the device to create possible occlusion to account for failed manipulation cases.

posed new part detection and gap detection methods by using and training state-of-the-art deep learning networks. We showed on the use-case of a HDD that the pipeline can achieve a high detection and localization performance, enabling a robot to interact with the device with sufficient precision. The overall process still make some errors $(80 \%$ AP for screw detection, $24 \%$ error for gap detection) but we do not see this as a limitation: such performance given the variability of brands, models and state of the device is acceptable; the goal of this pipeline is to be used in a more complex system were perception inaccuracies are handled based on action outcome. To the best of our knowledge, this is the first approach to provide an adaptable visual method to analyse a whole device in contrast to most of the related work, which focuses on detecting specific parts or properties of the mate- rial. This scheme is designed to provide robots with robust analysis capabilities and open new grounds for automated disassembly and recycling of End-Of-Life electronic devices.

One challenge of applying vision methods with off-the-shelf cameras to such a domain lies in the presence of shiny and reflective parts. These reduce the quality of the point-cloud introducing potential errors in the position estimation and the gap detection. Our system nevertheless provides a relevant analysis of the hard drive state but the lighting conditions are a potential failure parameter and should thus be controlled. On the other hand, the method is weakly affected by damages to the device in this use case.

The current need for top-view inputs of the device limits the ability of the system to analyse all potential scenes. It can however be overcome by training with more varied data including side views of the device (for the part detection and screw detection modules). For the gap detection, the methods relies on a perceived depth difference so the gaps can be detected in any position of the device (but are easier to detect when they are aligned with the stereo camera).

Gap detection can directly be adapted to a new device as it requires no training data and can be universally found in any device. The screws used here are only of Torx 8 type and different types of screws should be included into the training dataset if a different device is to be disassembled. However, this is not a time consuming task, thanks to the convenient data collection mode of the screw detector (Yildiz and Wörgötter, 2019). The part recognition is the least directly generalizable component and requires collection, annotation and retraining for other class of devices. However, the HDD use case offers a wide range of parts that can also be found in other devices (e.g. PCB, lid and covers), even if they have different appearances. We will evaluate to which extend the current system performs well on a set of new devices in future work.

\section{ACKNOWLEDGMENTS}

The research leading to these results has received funding from the European Union's Horizon 2020 research and innovation programme under grant agreement no. 731761, IMAGINE. We would like to also express our gratitude to Maik Bergamos and Electrocycling $\mathrm{GmbH}^{4}$ for the data and hardware used in this work as well as their expertise on the recycling domain.

\footnotetext{
${ }^{4} \mathrm{http} / / /$ www.electrocycling.de
} 


\section{REFERENCES}

Abadi, M., Barham, P., Chen, J., Chen, Z., Davis, A., Dean, J., Devin, M., Ghemawat, S., Irving, G., Isard, M., et al. (2016). Tensorflow: A system for largescale machine learning. In 12th \{USENIX $\}$ Symposium on Operating Systems Design and Implementation $(\{O S D I\} 16)$, pages 265-283.

Badrinarayanan, V., Kendall, A., and Cipolla, R. (2017). Segnet: A deep convolutional encoder-decoder architecture for image segmentation. IEEE transactions on pattern analysis and machine intelligence, 39(12):2481-2495.

Bailey-Van Kuren, M. (2002). Automated demanufacturing studies in detecting and destroying, threaded connections for processing electronic waste. In Conference Record 2002 IEEE International Symposium on Electronics and the Environment (Cat. No.02CH37273), pages 295-298.

Bdiwi, M., Rashid, A., and Putz, M. (2016). Autonomous disassembly of electric vehicle motors based on robot cognition. Proceedings - IEEE International Conference on Robotics and Automation, 2016-June(July):2500-2505.

Bi, Z. and Wang, L. (2010). Advances in 3d data acquisition and processing for industrial applications. Robotics and Computer-Integrated Manufacturing, 26(5):403 413.

Bisong, E. (2019). Google colaboratory. In Building Machine Learning and Deep Learning Models on Google Cloud Platform, pages 59-64. Springer.

Cai, Z. and Vasconcelos, N. (2018). Cascade r-cnn: Delving into high quality object detection. In Proceedings of the IEEE conference on computer vision and pattern recognition, pages 6154-6162.

Chen, C., Ma, L., Jia, Y., and Zuo, P. (2019). Kidney and tumor segmentation using modified 3d mask renn.

Chollet, F. (2017). Xception: Deep learning with depthwise separable convolutions. In Proceedings of the IEEE conference on computer vision and pattern recognition, pages 1251-1258.

Couteaux, V., Si-Mohamed, S., Nempont, O., Lefevre, T., Popoff, A., Pizaine, G., Villain, N., Bloch, I., Cotten, A., and Boussel, L. (2019). Automatic knee meniscus tear detection and orientation classification with mask-rcnn. Diagnostic and interventional imaging, 100(4):235-242.

Dai, J., He, K., and Sun, J. (2015). Boxsup: Exploiting bounding boxes to supervise convolutional networks for semantic segmentation. In 2015 IEEE International Conference on Computer Vision, ICCV 2015, Santiago, Chile, December 7-13, 2015, pages 16351643. IEEE Computer Society.

Dai, J., He, K., and Sun, J. (2016). Instance-aware semantic segmentation via multi-task network cascades. In Proceedings of the IEEE Conference on Computer Vision and Pattern Recognition, pages 3150-3158.

Duda, R. O. and Hart, P. E. (1971). Use of the hough transformation to detect lines and curves in pictures. Technical report, SRI Iinternational Menlo Park CA Artificial Intelligence Center.
Duflou, J. R., Seliger, G., Kara, S., Umeda, Y., Ometto, A., and Willems, B. (2008). Efficiency and feasibility of product disassembly: A case-based study. CIRP Annals, 57(2):583-600.

Dvornik, N., Shmelkov, K., Mairal, J., and Schmid, C. (2017). Blitznet: A real-time deep network for scene understanding. In Proceedings of the IEEE international conference on computer vision, pages 4154 4162.

Elsayed, A., Kongar, E., Gupta, S. M., and Sobh, T. (2012). A robotic-driven disassembly sequence generator for end-of-life electronic products. Journal of Intelligent and Robotic Systems: Theory and Applications, 68(1):43-52.

Fu, C.-Y., Shvets, M., and Berg, A. C. (2019). Retinamask: Learning to predict masks improves state-ofthe-art single-shot detection for free. arXiv preprint arXiv:1901.03353.

Ghiasi, G. and Fowlkes, C. C. (2016). Laplacian pyramid reconstruction and refinement for semantic segmentation. In European Conference on Computer Vision, pages 519-534. Springer.

Girshick, R. (2015). Fast r-cnn. In Proceedings of the IEEE international conference on computer vision, pages 1440-1448.

Girshick, R., Donahue, J., Darrell, T., and Malik, J. (2014). Rich feature hierarchies for accurate object detection and semantic segmentation. In Proceedings of the IEEE conference on computer vision and pattern recognition, pages 580-587.

He, K., Gkioxari, G., Dollár, P., and Girshick, R. (2017). Mask r-cnn. In Proceedings of the IEEE international conference on computer vision, pages 2961-2969.

Howard, A. G., Zhu, M., Chen, B., Kalenichenko, D., Wang, W., Weyand, T., Andreetto, M., and Adam, H. (2017). Mobilenets: Efficient convolutional neural networks for mobile vision applications. arXiv preprint arXiv:1704.04861.

Huang, G., Liu, Z., and Weinberger, K. Q. (2016). Densely connected convolutional networks. CoRR, abs/1608.06993.

Jahanian, A., Le, Q. H., Youcef-Toumi, K., and Tsetserukou, D. (2019). See the e-waste! training visual intelligence to see dense circuit boards for recycling. In Proceedings of the IEEE Conference on Computer Vision and Pattern Recognition Workshops, pages 0 0 .

Johnson, J. W. (2018). Adapting mask-renn for automatic nucleus segmentation. arXiv preprint arXiv:1805.00500.

Joulin, A., van der Maaten, L., Jabri, A., and Vasilache, N. (2016). Learning visual features from large weakly supervised data. In Computer Vision - ECCV 2016, pages $67-84$.

Karlsson, B. and Järrhed, J.-O. (2000). Recycling of electrical motors by automatic disassembly. Measurement Science and Technology, 11(4):350-357.

Khoreva, A., Benenson, R., Hosang, J., Hein, M., and Schiele, B. (2017). Simple does it: Weakly supervised instance and semantic segmentation. In 2017 IEEE Conference on Computer Vision and Pattern Recognition (CVPR), pages 1665-1674. 
Krizhevsky, A., Sutskever, I., and Hinton, G. E. (2012). Imagenet classification with deep convolutional neural networks. In Advances in neural information processing systems, pages 1097-1105.

Li, Y., Qi, H., Dai, J., Ji, X., and Wei, Y. (2017). Fully convolutional instance-aware semantic segmentation. In Proceedings of the IEEE Conference on Computer Vision and Pattern Recognition, pages 2359-2367.

Lin, D., Shen, D., Shen, S., Ji, Y., Lischinski, D., CohenOr, D., and Huang, H. (2019). Zigzagnet: Fusing topdown and bottom-up context for object segmentation. In Proceedings of the IEEE Conference on Computer Vision and Pattern Recognition, pages 7490-7499.

Lin, G., Milan, A., Shen, C., and Reid, I. (2017a). Refinenet: Multi-path refinement networks for highresolution semantic segmentation. In Proceedings of the IEEE conference on computer vision and pattern recognition, pages 1925-1934.

Lin, T.-Y., Dollár, P., Girshick, R., He, K., Hariharan, B., and Belongie, S. (2017b). Feature pyramid networks for object detection. In Proceedings of the IEEE Conference on Computer Vision and Pattern Recognition, pages 2117-2125.

Lin, T.-Y., Maire, M., Belongie, S., Hays, J., Perona, P., Ramanan, D., Dollár, P., and Zitnick, C. L. (2014). Microsoft coco: Common objects in context. In European conference on computer vision, pages 740-755. Springer.

Liu, S., Qi, L., Qin, H., Shi, J., and Jia, J. (2018). Path aggregation network for instance segmentation. In Proceedings of the IEEE Conference on Computer Vision and Pattern Recognition, pages 8759-8768.

Liu, Y., Wang, Y., Wang, S., Liang, T., Zhao, Q., Tang, Z., and Ling, H. (2019). Cbnet: A novel composite backbone network architecture for object detection. arXiv preprint arXiv:1909.03625.

Long, J., Shelhamer, E., and Darrell, T. (2015). Fully convolutional networks for semantic segmentation. In Proceedings of the IEEE conference on computer vision and pattern recognition, pages 3431-3440.

Malamas, E. N., Petrakis, E. G., Zervakis, M., Petit, L., and Legat, J.-D. (2003). A survey on industrial vision systems, applications and tools. Image and Vision Computing, 21(2): 171 - 188 .

Massari, S. and Ruberti, M. (2013). Rare earth elements as critical raw materials: Focus on international markets and future strategies. Resources Policy, 38(1):36-43.

Otsu, N. (1979). A threshold selection method from graylevel histograms. IEEE transactions on systems, man, and cybernetics, 9(1):62-66.

Pinheiro, P. O., Lin, T.-Y., Collobert, R., and Dollár, P. (2016). Learning to refine object segments. In $E u$ ropean Conference on Computer Vision, pages 75-91. Springer.

Pomares, J., Puente, S., Torres, F., Candelas, F., and Gil, P. (2004). Virtual disassembly of products based on geometric models. Computers in Industry, 55(1):114.

Ren, S., He, K., Girshick, R., and Sun, J. (2015). Faster r-cnn: Towards real-time object detection with region proposal networks. In Advances in neural information processing systems, pages 91-99.
Ronneberger, O., Fischer, P., and Brox, T. (2015). U-net: Convolutional networks for biomedical image segmentation. In International Conference on Medical image computing and computer-assisted intervention, pages 234-241. Springer.

Rusu, R. B., Marton, Z. C., Blodow, N., Dolha, M., and Beetz, M. (2008). Towards 3D Point Cloud Based Object Maps for Household Environments. Robotics and Autonomous Systems Journal (Special Issue on Semantic Knowledge in Robotics), 56(11):927-941.

Sandler, M., Howard, A., Zhu, M., Zhmoginov, A., and Chen, L.-C. (2018). Mobilenetv2: Inverted residuals and linear bottlenecks. In Proceedings of the IEEE conference on computer vision and pattern recognition, pages 4510-4520.

Szegedy, C., Vanhoucke, V., Ioffe, S., Shlens, J., and Wojna, Z. (2016). Rethinking the inception architecture for computer vision. In Proceedings of the IEEE conference on computer vision and pattern recognition, pages $2818-2826$.

Tabuchi, H. (2010). Japan recycles minerals from used electronics. New York Times, 4

Tonko, M. and Nagel, H.-H. (2000). Model-based stereotracking of non-polyhedral objects for automatic disassembly experiments. International Journal of Computer Vision, 37(1):99-118.

Torres, F., Puente, S., and Aracil, R. (2003). Disassembly planning based on precedence relations among assemblies. International Journal of Advanced Manufacturing Technology, 21.

Ukida, H. (2007). Visual defect inspection of rotating screw heads. In SICE Annual Conference 2007, pages 14781483. IEEE.

Wegener, K., Chen, W. H., Dietrich, F., Dröder, K., and Kara, S. (2015). Robot assisted disassembly for the recycling of electric vehicle batteries. Procedia CIRP, 29:716-721.

Weigl-Seitz, A., Hohm, K., Seitz, M., and Tolle, H. (2006). On strategies and solutions for automated disassembly of electronic devices. International Journal of Advanced Manufacturing Technology, 30(5-6):561-573.

Xie, S., Girshick, R., Dollár, P., Tu, Z., and He, K. (2017). Aggregated residual transformations for deep neural networks. In Proceedings of the IEEE conference on computer vision and pattern recognition, pages 14921500.

Xie, S. Q., Cheng, D., Wong, S., and Haemmerle, E. (2008). Three-dimensional object recognition system for enhancing the intelligence of a KUKA robot. International Journal of Advanced Manufacturing Technology, 38(7-8):822-839.

Yildiz, E. and Wörgötter, F. (2019). DCNN-Based Screw Detection for Automated Disassembly Processes. Proceedings of the 15th International Conference on Signal Image Technology \& Internet based Systems.

Zhang, M., Zhou, Y., Zhao, J., Man, Y., Liu, B., and Yao, R. (2019). A survey of semi- and weakly supervised semantic segmentation of images. Artificial Intelligence Review. 